\title{
Preparation and Characteristics of Single Layer Oxide Thin Film
}

\author{
Xiaolong Zhu ${ }^{\text {a }}$, Jun Xiao ${ }^{\text {b }}$ \\ School of Southwest University for Nationalities, Chengdu, 610041, China \\ a90357703@qq.com, bxiaojun@swun.cn
}

Keywords: oxide film, optical constants, surface roughness, water absorption peak.

\begin{abstract}
This This $\mathrm{Al}_{2} \mathrm{O}_{3}, \mathrm{SiO}_{2}, \mathrm{Ta}_{2} \mathrm{O}_{5}, \mathrm{Nb}_{2} \mathrm{O}_{5}, \mathrm{TiO}_{2}$ single layer oxide thin film are prepared by using ion beam sputtering deposition technology. The oxide films infrared optical constants are fitted by infrared variable elliptic polarization Angle spectrum instrument (IR - VASE), the results show that the fitting optical constants $\mathrm{n}, \mathrm{k}$ in line with the material properties. The oxide thin film surface roughness are analyzed by AFM (Nanosurf Easysc -2), and research findings show the compact structure of oxide film and RMS of surface roughness in $0.1 \mathrm{~nm}$, which scattering is smaller.
\end{abstract}

\section{Introduction}

The main working band of infrared detector and chemical laser are in the infrared 2-6 $\mu \mathrm{m}$ band. The application of the optical thin film material is mainly fluoride, sulfide and selenite soft membrane materials in infrared wavelengths. Oxide has been widely used in visible near infrared wave band with the superior mechanical properties and environmental stability, and prepared for high performance optical thin film. But research and application of infrared wavelengths in oxide are rarely reported. It is of great significant to widen the application range of the oxide film, and solve the traditional infrared thin film material environmental stability and poor adhesion.

\section{Experimental}

Experiment coating equipment of ion beam sputtering coating machine produced by United States Veeco company .Coating target materials and the purity are respectively: $\mathrm{SiO}_{2}$ (99.999\%), $\mathrm{Al}$ (99.999\%), Ta (99.99\%), $\mathrm{Nb}$ (99.95\%), $\mathrm{TiO}_{2}$ (99.99\%), target size is about $35.5 \mathrm{~cm}$ (14 inches). Experimental background of vacuum are under $5 \times 10^{-4} \mathrm{pa}$, the pressure in the process of coating remain at around $4.2 \times 10^{-2}$ pa.

\section{Results and Discussion}

\subsection{Thin film optical properties in IR}

The optical constant dispersion curve of Liquid water is shown in Figure 1.
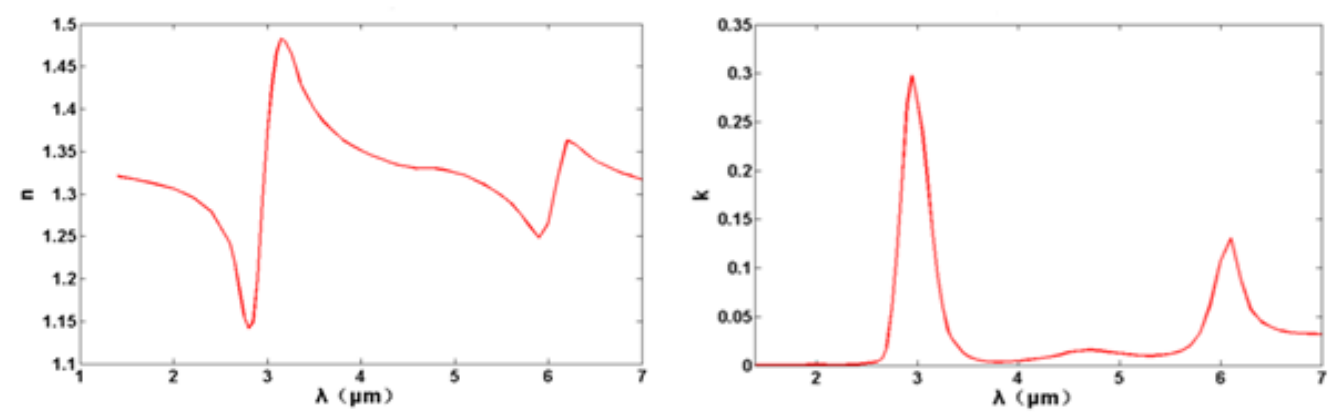

Figure. 1 optical constant dispersion curve of liquid water

It can be seen from the diagram, the water, there are two large absorption peak in the $2.94 \mu \mathrm{m}$ and 6.15 $\mu \mathrm{m}$ [1]. The film absorb moisture can lead to a dramatic absorption band in HF laser and be unavailable. Therefore, it is the key to effectively reduce the water absorption while preparation of infrared film. 
The oxide thin film infrared transmittance curves are tested by Spectrum GX Optical Fourier transform infrared spectrometer produced by PE Company. And results are shown in Figure. 2.
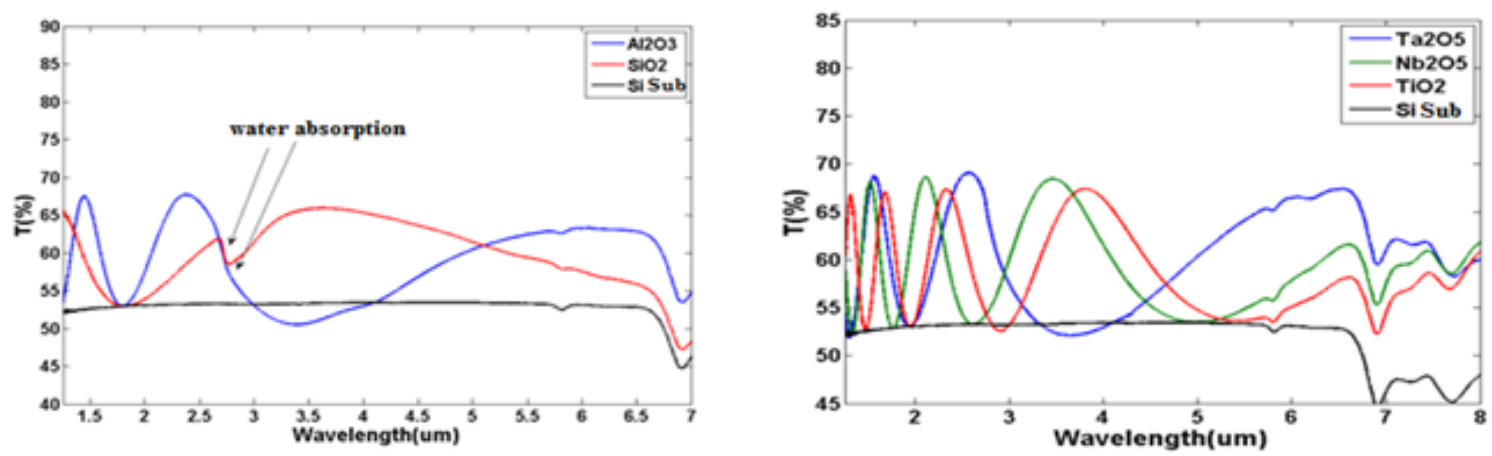

Figure. 2 oxide thin film infrared transmittance curves

From the figure, we can found that $\mathrm{Al} 2 \mathrm{O} 3$ and $\mathrm{SiO} 2$ as low refractive index material film shows obviously the water absorption near $2.9 \mu \mathrm{m}$, which cause its spectral curve concave [2]. For high refractive index materials, Ta2O5 also shows certain water absorption in $2.9 \mu \mathrm{m}$ and $6.2 \mu \mathrm{m}$ spectrum curve of the sag. But Nb2O5 and $\mathrm{TiO} 2$ do not appear obvious water absorption [3]. Analyzing the reasons of the water absorption may have the followings: 1 . when the vacuum pump to a certain extent, the $\mathrm{H} 2 \mathrm{O}$ is the residual gas in the vacuum chamber molecules and $\mathrm{H} 2 \mathrm{O}$ can be deposited on the substrate surface with thin film molecules while thin film deposition process; 2 . After completion of coating, the process of exhaust gas into the vacuum chamber, because of the film density less than 1 , it lead to the adsorption of water molecules within a small space [4]. 3. The target material itself may contain traces of $\mathrm{OH}$-, which were splashed and deposited in the substrate in the process of coating with target atoms.

The infrared optical constants of several oxide film are tested by infrared variable elliptic polarization Angle spectrum instrument (IR-VASE), and use a single Gaussian absorption model to fit the water absorption peak. The dispersion curves as shown in Figure. 3.
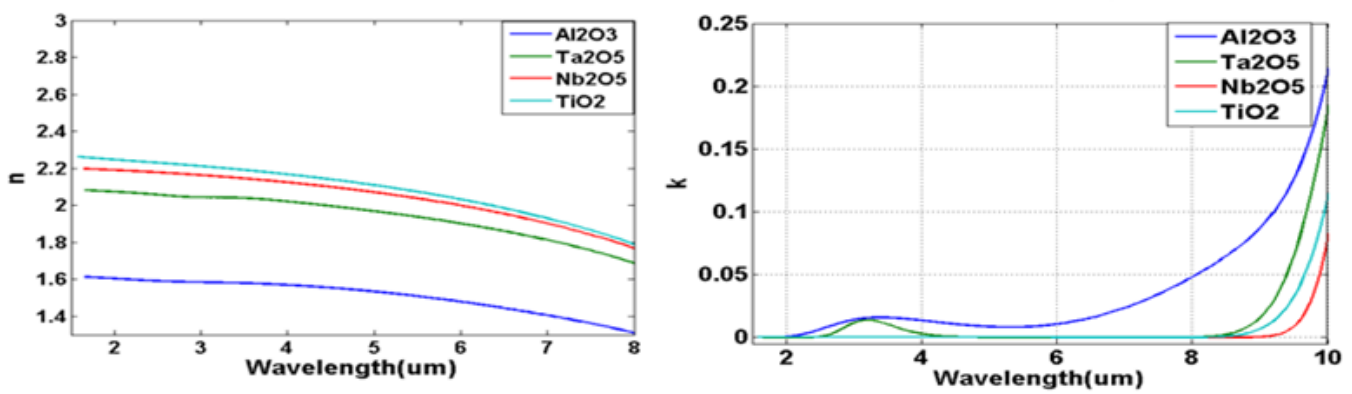

Figure. 3 n \& k dispersion curves of oxide film

Table2 Thin film preparation process parameters

\begin{tabular}{ccccc}
\hline & $\mathrm{n}(2.7 \mu \mathrm{m})$ & $\mathrm{k}(2.7 \mu \mathrm{m})$ & $\mathrm{n}(3.8 \mu \mathrm{m})$ & $\mathrm{k}(3.8 \mu \mathrm{m})$ \\
\hline $\mathrm{Al} 2 \mathrm{O} 3$ & 1.29 & $9 \times 10-3$ & 1.574 & 0.015 \\
$\mathrm{Ta} 2 \mathrm{O} 5$ & 2.05 & $3.5 \times 10-3$ & 2.03 & $5 \times 10-3$ \\
$\mathrm{Nb} 2 \mathrm{O} 5$ & 2.17 & 0 & 2.13 & 0 \\
$\mathrm{TiO} 2$ & 2.22 & 0 & 2.18 & 0 \\
\hline
\end{tabular}


From the refractive index of dispersion curve, the refractive index size order: TiO2 > Nb2O5 > Ta2O5> Al2O3 [5]. From extinction coefficient curve, Al2O3 and Ta2O5 film both shows the emergence of a large absorption peak near the $3 \mu \mathrm{m}$, so it proves the water absorption existed. Nb2O5 and TiO2 do not appear absorption in the absorption peak, which means the water absorption is small and hard to fit by elliptic partial instrument [6]. Thin film optical constants are presented in table 2 at $2.7 \mu \mathrm{m}$ and $3.8 \mu \mathrm{m}$.

\subsection{Thin film of the surface roughness and scattering}

Adopt the Nanosurf company Easysc - 2 AFM measured thin film surface roughness .The results shows in Figure. 4.

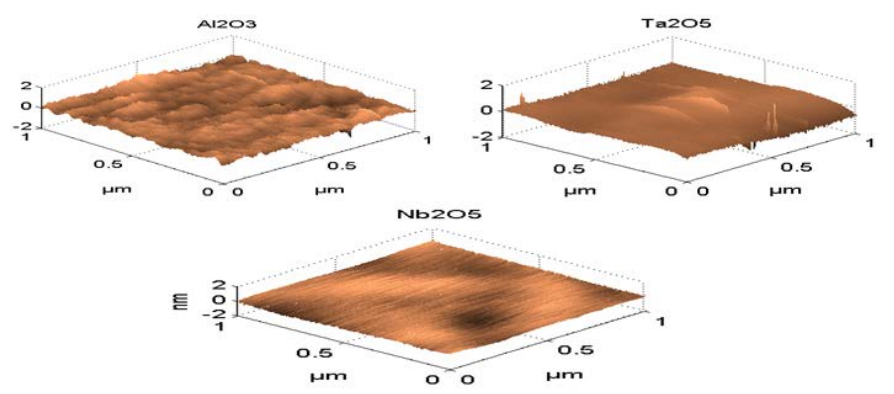

Figure. 4 Thin film of the AFM surface micro topography

According to the test data, we calculating the root mean square roughness by equation of the total single surface scattering (TIS) and root mean square (RMS) roughness on the surface [7]

$$
T I S=R_{0}\left(\frac{4 \pi \sigma n_{0}}{\lambda}\right)^{2}+T_{0}\left[\frac{2 \pi \sigma}{\lambda}\left(n_{f}-n_{0}\right)^{2}\right]
$$

In equation 1 , parameter R0, T0 mean the reflectivity and transmittance of film surface. $\mathrm{n} 0$ and $\mathrm{nf}$ mean the refractive index for incident medium and thin film, and the $\sigma$ for RMS roughness of the interface[8]. To calculate the TIS of Al2O3, Ta2O5 and Nb2O5 films in $2.7 \mu \mathrm{m}$ and $3.8 \mu \mathrm{m}$, and the consequences list in Table 3 below:

Table 3 Thin films root mean square roughness scattering and total integral value

\begin{tabular}{lccl}
\hline & $\mathrm{Al}_{2} \mathrm{O}_{3}$ & $\mathrm{Ta}_{2} \mathrm{O}_{5}$ & $\mathrm{Nb}_{2} \mathrm{O}_{5}$ \\
\hline $\mathrm{RMS} / \mathrm{nm}$ & 0.175 & 0.200 & 0.121 \\
$\mathrm{TIS}(\lambda=2.7 \mu \mathrm{m})$ & $8.9 \times 10^{-8}$ & $2.97 \times 10^{-7}$ & $1.25 \times 10^{-7}$ \\
$\mathrm{TIS}(\lambda=3.8 \mu \mathrm{m})$ & $4.5 \times 10^{-8}$ & $1.5 \times 10^{-7}$ & $6.3 \times 10^{-8}$ \\
\hline
\end{tabular}

As we can see from the Table, The preparation of ion beam sputtering oxide films have a low RMS and are in order of magnitude $0.1 \mathrm{~nm}$. The corresponding total integral scattering is very small, which to the point of can be ignored [9].

\section{Summary}

The preparation of single layer $\mathrm{Al} 2 \mathrm{O} 3$, SiO2, Ta2O5, Nb2O5 and $\mathrm{TiO} 2$ thin film with the ion beam sputtering method. Using infrared spectrometer and infrared elliptic partial instrument measure the thin film spectrum and optical constants respectively, And contrast analyze water absorption with different thin film materials, the results showed $\mathrm{Nb} 2 \mathrm{O} 5$ and $\mathrm{TiO} 2$ thin films with smaller water absorption. Study Al2O3, Ta2O5, Nb2O5 three kinds of thin film surface microstructure, roughness and environmental stability. The results showed that ion beam sputtering in preparation of the characteristics of thin film surface was smooth and had low roughness. The basic research had a significance guiding for the future design of the Multilayer films [10]. 


\section{Acknowledgment}

This research is supported by the southwestern ethnic university innovative research project. Scientific research item NO.CX2015SZ111

\section{Reference}

[1].George M Hale,Marvin R Querry. Optical constants of water in the $200 \mathrm{~nm}$ to $200 \mu \mathrm{m}$ region[J]. Applied Optics,1973,12(3):555-563.

[2].Eda Cetinorgu, Bill Baloukas, Oleg Zabeida, et al. Mechanical and thermoelastic characteristics of optical thin films deposited by dual ion beam sputtering[J]. Applied Optics, 2009, 48 (23):4536-4544.

[3].J Nguyen,M Razeghi.Techniques for High-Quality SiO2 Films[C].Proc of SPIE,2007,6479: 1K-1:8.

[4].Drik Poelman,philippe Frederic Smet. Methods for the determination of the optical constants of thin films from single transmission measurements:a critical review[J]. Appl.Phys,2003,36: 1850 $-1857$.

[5].Cheng-Chung Lee,Jin-Cherng Hsu,Daw-Hang Wong. The characteristics of some metallic oxides prepared in high vacuum by ion beam sputtering[J]. Applied Surface Science, 2001,171:151-156.

[6].Zhang long,Chen lei,Fan Youyu. development and front application of infrared glass material [J]. Journal of the optical,2011,31(9):1-9.

[7].Hong Dongmei,Yue Wei. Development of the infrared laser optical thin film [J]. Laser and infrared,2006,36(12):1157-1159.

[8].Martínez F L, Luque M T, Gandía J J, et al. Optical properties and structure of HfO2 thin films grown by high pressure reactive sputtering [J]. J. Phys. D: Appl. Phys(S0022-3727), 2007, 40:5256-5265.

[9].Li Jing ping,Fang Ming,He Dongbo.Model of stress evolution in polycrystalline oxide and composite thin films[J]Acta Optica Sinica,2012,32(10):1031004

[10].Laha A, Fissel A, Bugiel E. Epitaxial mult-i component rare earth oxide for high-kapplication[J]. Thin Solid Films, 2007,515:6512. 